\title{
Mental health and wellbeing support in sport
}

Tips for selecting a provider

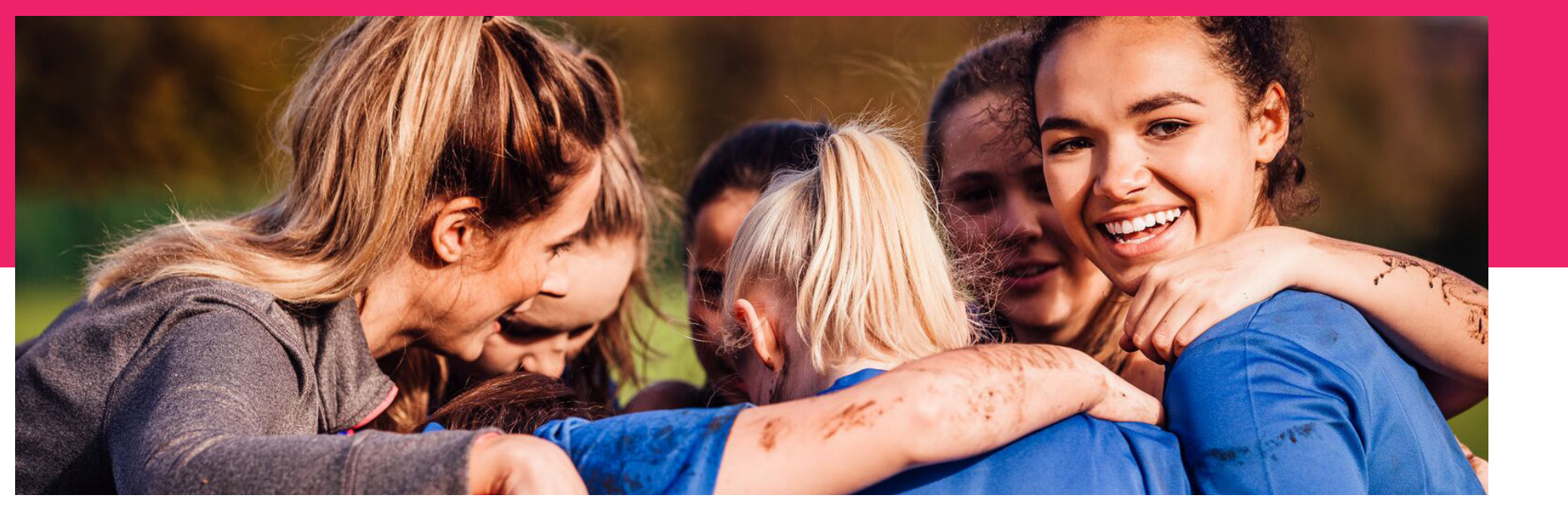

Is your sports organisation planning to provide mental health and wellbeing programs to participants?

Have you engaged mental health and wellbeing providers to deliver training or health promotion programs in the past?

If the answer is yes, thank you, your commitment to the mental health and wellbeing (MHW) of your community is vital. And, selecting the right provider will enable you to deliver the best possible program to your members.

A new review of MHW support in community sport by VicHealth and Monash University shows there is a wide range of providers in the sector.

However, whilst these organisations play a valuable role in developing positive MHW, the sector remains largely unregulated with providers offering a multitude of differing services of varying quality. This can be challenging for sporting clubs, leagues and associations to navigate.

This summary provides recommendations to help sporting clubs, leagues and associations to select a provider offering safe and effective programs.

\section{Why support for positive mental health and wellbeing is important}

Community sport offers an ideal space to support MHW within local communities, particularly for young people. It often provides a safe space and a trusted network where people feel socially connected.

High numbers of young people continue to participate in organised sport, meaning that clubs can provide an important context outside of schools where positive messages, MHW education and guidance regarding support services can be provided.

\section{What mental health and wellbeing programs are available?}

There are rapidly increasing numbers of organisations offering MHW support services to community sports clubs in Victoria. These can be individuals, businesses, not-for-profit organisations, or community agencies that deliver programs and provide resources to sporting clubs.

The review of these organisations found they offered a range of services and approaches, including:

- guest talks to club members

- education programs

- strategies to support mental health and wellbeing

- support pathways.

Clubs also have a duty of care to ensure that the MHW providers they are partnering with are safe and do no harm to the mental health or wellbeing of people at the club.

Navigating the range of programs for sporting clubs, leagues and associations can be challenging. The following questions are designed to guide your organisation through selection of an appropriate provider. 


\section{What to consider when engaging a mental health and wellbeing provider}
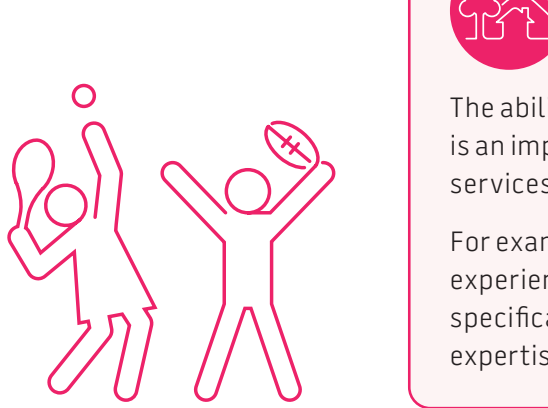

\section{What expertise in MHW in sport does the provider organisation have?}

The ability to tailor content and adjust to your specific context is an important indicator of quality programs. Consider what services you need and match this with provider expertise.

For example, if you are seeking to support young people, what experience do providers have with delivering MHW programs specifically for young people? If clubs are in rural areas, what expertise do they have in rural MHW issues and support systems?

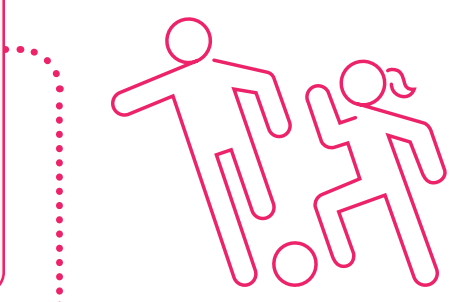

Does the provider seek to support ongoing cultural change in your club?

Whole of club approaches, where engagement with MHW providers is one part of a comprehensive mental health framework adopted by the club, leads to the development of positive and informed community sport cultures. Cultural change requires everyone to have a role in providing mentally healthy club environments.

Club leaders are integral to show commitment and action that champions positive and safe MHW approaches. Quality programs will engage club leaders (presidents, committee, head coaches, parents) in their approach and require them to make a commitment to the programs and strategies.

\section{What approach or model does the organisation use?}

The most effective MHW programs offer multiple points for follow up contact, rather than one-off awareness raising events, which can be harmful if they are not part of a whole of club approach. This could include offering a series of education sessions, working with the committee to implement systemic club strategies, providing support resources and offering clear next steps for clubs and members to gain further support and lasting benefit.

It is important to confirm the provider has an escalation policy in place to support any mental health concerns that might be raised by members. Clubs should also have an escalation policy to support members after the program. Consider having 'where to find help' information and include links to local support, such as headspace, primary mental health networks, community health organisations or GPs.

How long has the provider worked within the community sport sector?

This is not to dismiss newer organisations, but ongoing delivery over a sustained period suggests a trusted and refined program. If an organisation is relatively new to community sport, have they been working in other sectors previously such as schools or workplaces?

\section{Are mental health professionals} involved in developing programs?

Quality programs will have ongoing input from mental health professionals (such as psychologists, social workers or mental health nurses). Appropriately qualified specialists should be involved in the design, development and ongoing refinement to ensure programs are up to date with the latest research informed approaches. Personal stories of lived experience are important to reduce stigma. But, without professional input to sessions, an individual's story may not represent the complexity of MHW issues.

Who are the program facilitators and what are their qualifications?

What training have the program facilitators received? Quality programs will provide training for their facilitators and ensure they engage in ongoing professional development. Facilitators who have pre-existing skill sets and qualifications are well placed to deliver MHW interventions. These include social workers, youth workers, teachers, mental health nurses, psychologists or peer support youth trained in mental health first aid. Lived experience stories are most effective when those delivering them also undertake relevant training and ongoing professional development.

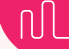

What evidence can the organisation provide that documents their program's impact?

Ideally providers should be evaluating their programs and can provide evidence of achieving their stated outcomes. If for example, the organisation claims their program enhances community coaches' knowledge of supporting young people with a mental health condition, what evidence can they provide that their program does this? Are they willing to share this evidence?

Check if the provider has partnerships with external research institutions, such as universities, when assessing the rigour of a program's evaluation. Don't rely solely on testimonials, word of mouth or participant surveys delivered by the providers to determine the effectiveness and safety of a program. 


\section{Useful resources}

You will find a list of providers offering mental health and wellbeing support to community sports clubs in Victoria here. Please note that VicHealth does not endorse any providers on this list.

\section{- VicHealth's blog}

Ten go-to mental health organisations for young people

\section{- Reboot Sport's blog}

4 Steps to Reboot Mental Health through Sport

\section{- AFL and Orygen}

What works for mental health in sporting teams

\section{- Head to Health}

https://headtohealth.gov.au/

\section{Get in contact}

VicHealth is partnering with VicSport to share this work with the sport sector. If you would like to ask a question or share your experience, please get in contact via admin@vicsport.com.au

\section{Acknowledgements}

We would like to acknowledge the team from Monash University who carried out this research:

Justen O'Connor

Ruth Jeanes

Christine Grové

Karen Lambert

Nadia Bevan

Hayley Truskewycz

Victorian Health Promotion Foundation P0 Box 154 Carlton South Victoria 3053 Australia

$T+61396671333 F+61396671375$

vichealth@vichealth.vic.gov.au

vichealth.vic.gov.au twitter.com/vichealth facebook.com/vichealth

VicHealth acknowledges the support of the Victorian Government.

(C) VicHealth 2020

December 2020

https://doi.org/10.37309/2020.PA1004 